\title{
Spectroscopic Analyses of the Noncovalent Self-Assembly of Cyanines upon Various Nucleic Acid Scaffolds
}

\author{
Komandoor E. Achyuthan, ${ }^{* \dagger}$ Jaime L. McClain,* Zhijun Zhou,** David G. WhitTen,** \\ and Darren W. BRANCH* \\ *Biosensors and Nanomaterials Department, Sandia National Laboratories, Albuquerque, NM, USA \\ **Department of Chemical and Nuclear Engineering, University of New Mexico, Albuquerque, NM, USA
}

\begin{abstract}
We utilized self-assembly of cyanine chromophores to study the conformational changes in various types of nucleic acid scaffolds: single and double stranded DNA, linear or circular DNA and RNA. We identified a chromophore that became highly fluorescent after aggregating upon nucleic acids. Fluorescence from the aggregate was instantaneous after selfassembly. Temporal emission profiles displayed a biphasic trend demonstrating kinetic dependence for assembly and disassembly. Absorption spectra of the aggregate showed a red-shifted "shoulder" peak indicative of J-aggregate. Fluorescence from J-aggregates was also red-shifted. We utilized cyanine self-assembly to quantize various nucleic acids. The limits of detection and quantization for $\phi$ X174 DNA were 3 and $9 \mathrm{fmol}$, respectively. We similarly determined the sensitivity for various nucleic acids and established the optimum conditions for self-assembly. Collectively, the effects of methanol, salt, and full width at half maximum for cyanine fluorescence on DNA or carboxymethylamylose scaffolds, all suggested noncovalent, electrostatic, and hydrophobic forces were involved in supramolecular self-assembly. Our results facilitate a better understanding of supramolecular self-assembly.
\end{abstract}

(Received July 8, 2008; Accepted September 22, 2008; Published April 10, 2009)

Noncovalent forces are involved in the supramolecular selfassembly. ${ }^{1-4}$ We previously showed that a class of chromophores known as cyanines were capable of spontaneously selfassembling upon a variety of biopolymers including

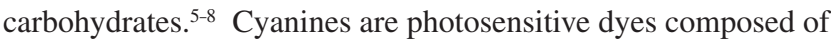
two quaternized, nitrogen-containing, and heterocyclic ring structures, that are linked by a polymethine bridge. ${ }^{9}$ Cyanines are characterized by high molar absorptivity, low intrinsic fluorescence and large fluorescence enhancement following self-assembly upon various templates. Supramolecular selfassembly involves reversible, noncovalent, and electrostatic (coulombic) interactions including hydrogen and/or charge bonding, van der Waals and hydrophobic forces. Cyanine selfassembly is sometimes accompanied by strong, sharp and bathochromic fluorescence, characteristic of J-aggregates. ${ }^{10}$ Cyanines are also capable of forming hypsochromic, nonfluorescent or very weakly fluorescent H-aggregates. ${ }^{10}$ Controlling and tuning the supramolecular self-assembly processes will offer insights to enable programmability to achieve desired functional nanomaterials. We therefore further investigated the self-assembling of cyanines from our molecular "library" in order to identify a chromophore that was capable of forming intensely fluorescent J-aggregates.

Our objectives were: 1) Identify cyanine chromophores from our molecular library that would be a sensitive probe for nucleic acids; 2) Correlate the form-function aspects of cyanine chemistry to self-assembly on nucleic acid scaffolds; 3) Compare the detection limits of single-stranded viral DNA, double-stranded viral DNA, double-stranded genomic DNA, and RNA, using self-assembly; 4) Optimize detection conditions for nucleic acids based upon supramolecular self-assembly

$\dagger$ To whom correspondence should be addressed.

E-mail: kachyut@sandia.gov including the stability of the nucleic acids-cyanine complex; and 5) Demonstrate supramolecular self-assembly processes were involved in the aggregation of the cyanine upon nucleic acid scaffolds. Results from these investigations are described in this paper.

\section{Experimental}

\section{Reagents and chemicals}

The chemical structures of various cyanines from our molecular "library"5,11 are shown in Fig. 1. Stock solutions of cyanines were prepared in methanol and stored refrigerated. Single stranded, circular, $\phi X 174$ virion DNA (5386 bases, $M_{\mathrm{r}}=$ $\left.1.7 \times 10^{6} \mathrm{Da}\right)$ from $\phi \mathrm{X} 174$ am 3 cs70 bacteriophage was purchased from New England Biolabs (Ipswich, MA). Greater than $85 \%$ of the DNA molecules were circular. The double stranded bacteriophage $\lambda$ DNA composed of $48502 \mathrm{bp}\left(M_{\mathrm{r}}=3.2\right.$ $\times 10^{7} \mathrm{Da}$ ) was from Promega (Madison, WI). Unsheared Escherichia coli (E. coli) genomic DNA with an average size of $16 \mathrm{~kb}\left(M_{\mathrm{r}}=1.4 \times 10^{7} \mathrm{Da}\right)$ was purchased from Sigma Aldrich (St. Louis, MO). The DNA had been purified by equilibrium buoyant density gradient ultracentrifugation in $\mathrm{CsCl}$ and agarose gel $(0.8 \%)$ electrophoresis was used for size determination. Genomic DNA was dissolved using TE buffer (10 mM Tris-HCl, $\mathrm{pH} 7.5,1 \mathrm{mM} \mathrm{NaCl}$ and $1 \mathrm{mM}$ EDTA). The DNA solution was stored in small aliquots at $-80^{\circ} \mathrm{C}$ and discarded after a single use. Saccharomyces cerevisiae ( $S$. cerevisiae, baker's yeast) RNA $\left(\sim 5.3 \mathrm{~kb}, 26 \mathrm{~S}, 17 \mathrm{~S}\right.$ and $\left.5.8 \mathrm{~S} ; M_{\mathrm{r}}=18 \times 10^{5} \mathrm{Da}\right)$ was from Sigma.

\section{Absorption spectra}

Absorption measurements were taken using quartz polymethylmethacrylate cuvette $(4 \mathrm{~mL})$, quartz cuvette $(600 \mu \mathrm{L})$ or polystyrene 96-microwell plates $(200 \mu \mathrm{L})$. Cyanine 

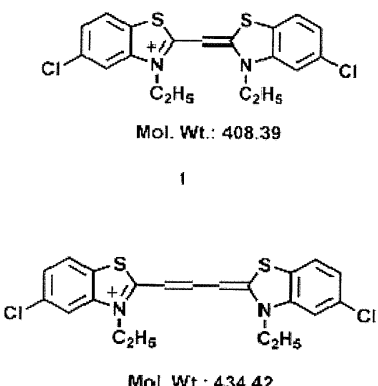

Mol. Wt.: 434.42

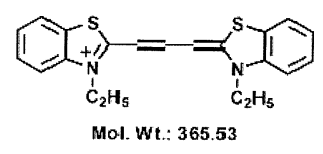

2

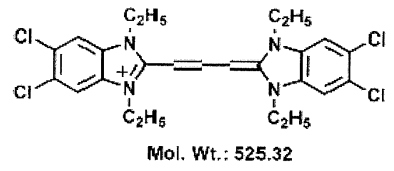

Fig. 1 Molecular structures of the cyanines. The molecular masses of the cyanines are shown in the figure. The four cyanines were obtained in the following salt forms $\mathrm{I}^{-}, \mathrm{Br}^{-}, \mathrm{PTS}^{-}$and $\mathrm{PTS}^{-}$and had the following molecular masses: 535.3, 445.5, 605.6 and 696.5, respectively. The absorption maxima (excitation wavelength, $\lambda$ ) were respectively, 435, 555, 561 and $514 \mathrm{~nm}$.

concentration was $10 \mu \mathrm{M}$ final. The nucleic acids were titrated into the cyanine solution in incremental amounts with gentle mixing before taking measurements. As negative controls, absorption sweeps of "vehicle" (i.e., 20\% methanol and 80\% water, v/v) and $10 \mu \mathrm{M}$ cyanine dissolved in vehicle were carried out. Measurements were taken using Molecular Devices (Sunnyvale, CA) instrument equipped with a cuvette holder or in the microwell plate reader. The volume change during titrations was approximately $7 \%$ of the total volume $\left(V_{\mathrm{t}}\right)$. Consequently, no corrections were made for volume changes.

\section{Fluorescence measurements}

The cyanines were diluted using a mixture of methanol-water $(20 / 80 \%, v / v)$. Nucleic acid solutions were also made in the same solvent. Fluorescence was measured at ambient temperature $\left(\sim 25^{\circ} \mathrm{C}\right)$ using a Mithras LB 940 microplate spectrofluorometer (Berthold Instruments, Oak Ridge, TN).,5 The filters for instrument were purchased from Chroma Technology (Rockingham, VT). In certain experiments, a monochromator-based microplate reader (Molecular Devices) was employed to measure excitation and emission. The reaction solutions $(200 \mu \mathrm{L})$ were dispensed in a 96-well white microplate (Perkin Elmer, Waltham, MA). Fluorescence from the Jaggregate was corrected for a small amount of intrinsic fluorescence of the cyanine monomer $(<10 \%$ of maximal fluorescence of J-aggregate). Certain measurements were taken using an ultra-micro quartz cuvette $(350 \mu \mathrm{L})$ with a FluoroMax2 spectrofluorometer (Horiba Jobin Yvon, Edison, NJ). Fluorescence spectra were taken under conditions similar to absorption measurements. Emission scans obtained using a quartz cuvette or microplate wells were similar thus assuring validity of the data. The volume change during titrations was $<10 \%$ of the total volume $\left(V_{\mathrm{t}}\right)$. Consequently, no corrections were made for volume changes.

\section{Methanol concentration effects upon self-assembly}

Each nucleic acid was tested at 25 and $50 \mathrm{ng}$. Fluorescence was measured immediately (1 to $2 \mathrm{~min}$ ) and after $30 \mathrm{~min}$ at $\sim 25^{\circ} \mathrm{C}$ to allow for self-assembly kinetics. Background fluorescence from $10 \mu \mathrm{M}$ cyanine mixed with increasing concentrations of methanol was determined. The type and concentration of the scaffold ( $\phi$ X174 DNA, $\lambda$ DNA, genomic DNA or RNA) and self-assembly kinetics influenced emission intensity. We therefore used changes in signal/background $(S / B)$ as an index of the effects of methanol upon cyanine selfassembly. ${ }^{12}$ We normalized the data as \% changes to $S / B$ and compared the effects of methanol upon the four nucleic acid scaffolds.

\section{Salt concentration effects upon self-assembly}

We examined the effects of $\mathrm{NaCl}$ on $\mathrm{J}$-aggregate fluorescence following cyanine self-assembly upon the nucleic acid scaffolds as described above for methanol.

Self assembly kinetics and stability of the self-assembled ensemble Either 5 or $10 \mu \mathrm{M}$ cyanine was collected upon $15 \mathrm{ng}$ of $\phi \mathrm{X} 174$ DNA, $15 \mathrm{ng} \lambda \mathrm{DNA}, 15 \mathrm{ng}$ E. coli genomic DNA or $25 \mathrm{ng} S$. cerevisiae RNA. Fluorescence emission was detected immediately (within $1 \mathrm{~min}$ ) after mixing cyanine and the nucleic acids, by exciting the samples at $427 \mathrm{~nm}$ and measuring the emission at $470 \mathrm{~nm}$. As negative control, fluorescence from six replicates of 5 and $10 \mu \mathrm{M}$ cyanine was also measured. Timed reactions were carried out in two separate ways. In the first, the same reaction wells were excited repeatedly over time and the fluorescence emission was measured. In the second method, dedicated reaction wells were maintained for each time point. In the latter technique, reaction mixtures were excited only once and the fluorescence emission was measured. Results are presented as percent changes to $S / B$ as described above.

\section{Data analysis}

All data were collected in triplicate. Results are average \pm standard deviation. Where not visible, error bars are masked within the symbol. Curve fitting was done using Kaleidagraph (Synergy Software, Reading, PA). The increase in fluorescence emission over various controls (cyanine "alone", nucleic acids "alone", vehicle "alone" and empty wells) was measured. The corrected signal was traced as relative fluorescence units (RFU) ${ }^{5,6}$ Limits of detection (LOD) and quantization (LOQ) were calculated as described previously. ${ }^{13}$

\section{Results and Discussion}

\section{Cyanine chemistry and spectral characterization}

We investigated the potential for J-aggregate formation of the various cyanines using nucleic acids as a template. The molecular structures and properties of four cyanines are shown in Fig. 1. Among these, the cyanines designated $\mathbf{1}$ and $\mathbf{4}$ were capable of forming fluorescent J-aggregates and we therefore focused on these chromophores. Since we had access to $\mathbf{2}$ and 4, we tested these as well. Until now, there is no clear understanding of the relationship between cyanine chemistry and J-aggregation potential. By testing the four cyanines with subtle differences in their structures, three out of four had the electron withdrawing chlorine substituent (Fig. 1), we hoped to better understand such relationships (vide infra). In order to characterize the fluorescence, we tested the cyanines using all combinations of the following filter sets: excitation filters: 320 , 355, 400, 485 and $650 \mathrm{~nm}$; emission filters: 400, 460, 480, 515, 530,535 and $685 \mathrm{~nm}$.

Cyanines $\mathbf{1}$ and $\mathbf{4}$ yielded moderate-to-high fluorescence as well as large $S / B$ after self-assembly upon the various nucleic acid scaffolds. Results for $\mathbf{1}$ were obtained using excitation and emission filters 400 and $460 \mathrm{~nm}$, respectively. These data are in accord with the absorption maximum for $\mathbf{1}$ (Fig. 2A). In the absence of scaffold, $\mathbf{1}$ had an absorption maximum at $425 \mathrm{~nm}$. Titration with $\phi \mathrm{X} 174$ DNA resulted in a progressive decrease of the absorption of $\mathbf{1}$ monomer at $425 \mathrm{~nm}$ along with the appearance of a new "shoulder" peak of between 445 and 480 

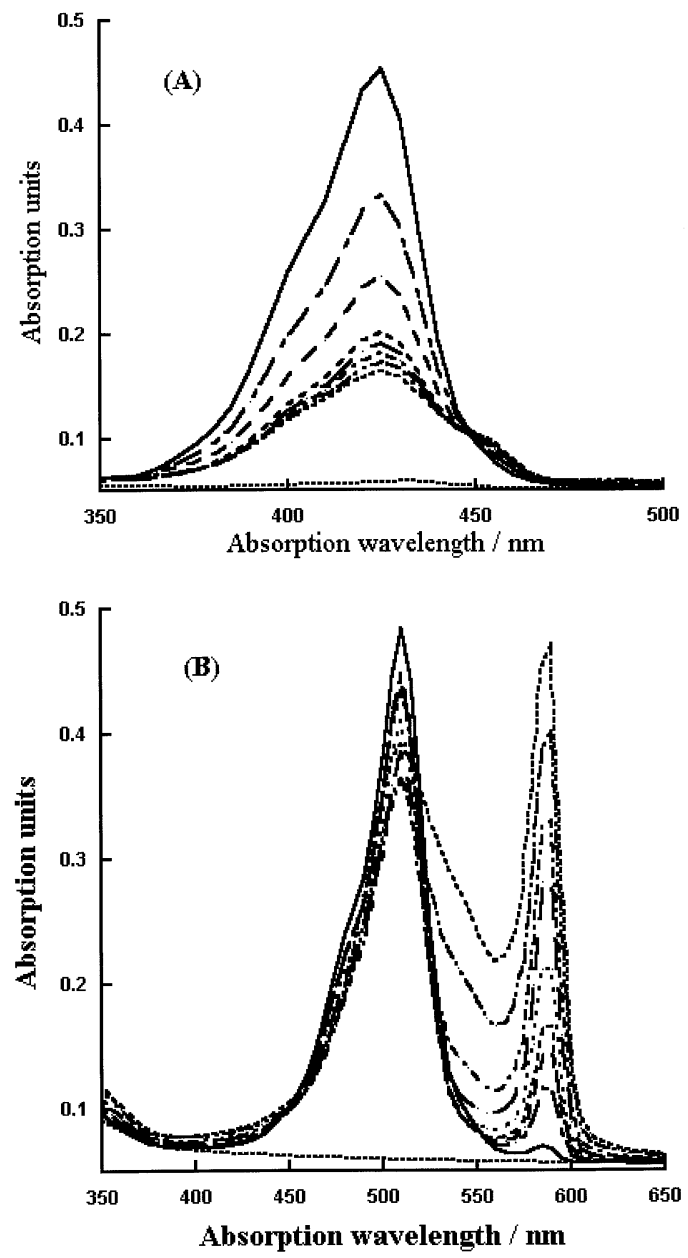

Fig. 2 (A) Absorption spectra of cyanine 1 titrated with increasing amounts of $\phi X 174$ DNA. Increasing amounts of the DNA were mixed with $10 \mu \mathrm{M}$ of $\mathbf{1}$. The tracings represent decreasing absorption intensities at $425 \mathrm{~nm}$ upon adding the following amounts of $\phi$ X174 DNA: $1.3,3.8,8.8,18.8,43.8,93.8,143.8$, and 310.8 ng DNA. The topmost solid line with the highest absorption represents the spectra of cyanine $\mathbf{1}$ in the absence of DNA. The tracings hugging the abscissa were from vehicle. (B) Absorption spectra of cyanine 4 titrated with increasing amounts of Saccharomyces cerevisiae (baker's yeast) RNA. Increasing amounts of the RNA were mixed with 10 $\mu \mathrm{M}$ of cyanine 4 in a total volume of $4 \mathrm{~mL}$ of a mixture of $20 \%$ methanol and $80 \%$ water $(\mathrm{v} / \mathrm{v})$. The tracings represent changes in the absorption profiles upon the addition of the following amounts of RNA: 90, 180, 270, 500, 1000, 2000, 5000 and 10000 ng RNA. Both the absorption profiles of cyanine $\mathbf{4}$ (left profiles) and the J-aggregate absorption profiles (right) are shown in this figure.

$\mathrm{nm}$, reflecting the bathochromicity that is characteristic of $\mathrm{J}$ aggregates (Fig. 2A). ${ }^{10}$ The absorption profiles of $\mathbf{1}$ with four different nucleic acid scaffolds were similar (data not shown). Cyanines $\mathbf{2}$ and $\mathbf{3}$ did not exhibit measurable fluorescence in the presence or absence of RNA. For these compounds there was also no development of the characteristic J-aggregate absorption at wavelengths red-shifted from the monomer. For $\mathbf{2}$ there was a monotonic decrease in absorption (ascribed to monomer) as increasing amounts of RNA were added; this observation is consistent with formation of a colloidal aggregate which may separate from the solution and either precipitate or adsorb on the cuvette walls. For 3, the monomer absorption at $561 \mathrm{~nm}$ decreased with addition of increasing amounts of RNA. There was also a small growth in absorption both to longer and shorter wavelengths which may be attributed to the development of an " $\mathrm{H}$ " aggregate; ${ }^{10}$ the presence of isosbestic points on either side of the cyanine monomer absorption suggests that the new species remains in solution during the addition of RNA. The absorption spectrum of $\mathbf{4}$ shows clear development of $\mathrm{J}$ aggregate upon addition of RNA (Fig. 2B); accompanying the change in absorption was an onset of fluorescence. The fluorescence occurred at similar wavelengths to those observed in other studies for the J-aggregate of $\mathbf{4}$ self-assembled on scaffolds such as Laponite clay. ${ }^{11}$

Cyanine dyes were shown to interact with DNA through several modes of binding (intercalation, groove binding and supramolecular self-assembly). ${ }^{8}$ Cyanine $\mathbf{2}$ has a structure that is chemically similar to $\mathbf{1}$, except for the methine bridge being two carbon atoms longer in $\mathbf{2}$ and the dichloro substituent of $\mathbf{1}$. Likewise, $\mathbf{1}$ and $\mathbf{3}$ are also identical except for the extra two carbons in the methine bridge of $\mathbf{3}$. Cyanine $\mathbf{4}$ has a similar conjugation length to $\mathbf{2}$ and $\mathbf{3}$ but differs in heterocycle structure and thus is not closely related structurally. These relatively small differences in chemical structures produced $>100 \mathrm{~nm}$ shifts in absorption maxima (excitation wavelength) and also resulted in the chromophores becoming either fluorescent $\mathrm{J}$ aggregates (1 and 4) or aggregates that were weakly or nonfluorescent (2 and $\mathbf{3}$ ). It was suggested that charged substituents on the dye could be used to control the overall structure of the Jaggregate on DNA templates. ${ }^{14}$ Since these are substituted cyanines (Fig. 1), J-aggregation may be favored due to steric and/or electrostatic interactions. ${ }^{8}$ From the absorption profiles (Fig. 2A), it appeared that there was only a small amount of conversion of $\mathbf{1}$ to J-aggregate. While $\mathbf{4}$ showed the most clearcut conversion to J-aggregate among the four dyes (Fig. 2B), the fluorescence of the J-aggregate was not nearly as strong as that of the various 1-nucleic acid complexes. Remarkably, the relatively small conversion of $\mathbf{1}$ to J-aggregates suggested by the small peak developing at $480 \mathrm{~nm}$ in the absorption spectra (Fig. 2A), compared to the much higher levels of J-aggregate formed when the same cyanine $\mathbf{1}$ self-assembled on a carbohydrate scaffold of carboxymethyl amylose,${ }^{15}$ nevertheless resulted in strong J-aggregate fluorescence for the 1-nucleic acid complex (Fig. 3). This suggested that the [1-nucleic acid] complex must have high quantum efficiency. Consequently, we focused on the details of the complexes formed between $\mathbf{1}$ and the different nucleic acids.

\section{Detector flexibility}

Experiments with a monochromator detector verified that the absorption maximum for $\mathbf{1}$ was $425 \mathrm{~nm}$ (Fig. 2A) and the fluorescence emission was maximal at $470 \mathrm{~nm}$ for the 1- $\phi \mathrm{X} 174$ DNA J-aggregate (Fig. 3). Similar results were obtained when emission spectra were collected using quartz cuvette and FluoroMax-2 fluorometer. Experiments involving 1 with the filter-based microplate reader were carried out using 400 and $460 \mathrm{~nm}$ excitation and emission filters, respectively. With the monochromator-detector, the excitation wavelength was set between 425 and $427 \mathrm{~nm}$, usually $426 \mathrm{~nm}$ (ex). Fluorescence emission was monitored at $470 \mathrm{~nm}$ (em). We similarly optimized the absorption and emission for $\mathbf{1}$ with each of the remaining nucleic acids: $\lambda \mathrm{DNA}, E$. coli $\mathrm{DNA}$ and $S$. cerevisiae RNA. Absorption and emission data for $\mathbf{1}$ and the various nucleic acid scaffolds were nearly identical to those shown in Figs. 2A and 3, respectively. With all scaffolds, the dominant absorption maximum for $\mathbf{1}$ monomer and $\mathbf{1}$ aggregating upon the nucleic acid templates was $425 \mathrm{~nm}$ along with the "shoulder" peak at $445-480 \mathrm{~nm}$ (Fig. 2A). The emission peak was broad in all cases, nearly $20 \mathrm{~nm}$ wide (463 to $486 \mathrm{~nm}$ ), with a peak at 


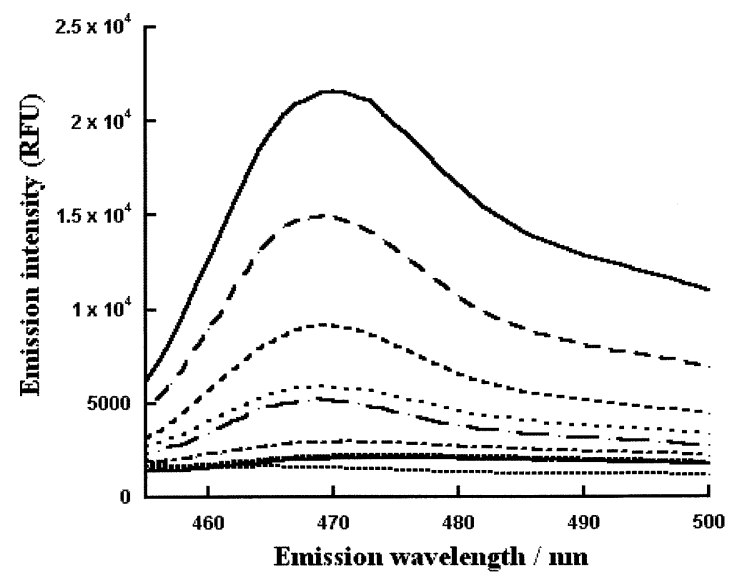

Fig. 3 Fluorescence emission spectra of cyanine 1 titrated with increasing amounts of $\phi \mathrm{X} 174$ DNA. Increasing amounts of DNA were mixed with $10 \mu \mathrm{M}$ of $\mathbf{1}$ in a total volume of $100 \mu \mathrm{L}$ of a mixture of $20 \%$ methanol and $80 \%$ water $(\mathrm{v} / \mathrm{v})$. The tracings represent the increases in the emission intensity upon adding the following amounts of $\phi X 174$ DNA: 1, 5, 10, 30, 50, 100, 200 and $300 \mathrm{ng}$ DNA. The topmost solid line represents fluorescence intensity in presence of $300 \mathrm{ng}$ DNA. The remaining tracings below represent the fluorescent intensities obtained with progressively decreasing amounts of DNA.

$470 \mathrm{~nm}$ (Fig. 3), representing a Stokes shift of $45 \mathrm{~nm}$ compared to 1 monomer peak absorption. At $100 \mathrm{ng}$ of each nucleic acid scaffold, the emission intensities of $\mathbf{1} \mathbf{J}$-aggregates were similar, being 1.5 to $3.0 \times 10^{4}$ RFU. System flexibility was thus demonstrated by generating similar data with the three different types of instruments (two different microplate readers and a fluorometer).

Evidence for supramolecular self-assembly of cyanine 1 on nucleic acid scaffolds

It was previously reported that methanol inhibited the aggregation of cyanine dyes by disrupting the electrostatic and hydrophobic interactions. ${ }^{14}$ Salt $(\mathrm{NaCl})$ was also found to influence the aggregation of cyanine dyes on DNA scaffolds in a concentration- and temperature-dependent manner. ${ }^{14}$ It is clear from Fig. 4, that $20 \%(\mathrm{v} / \mathrm{v})$ methanol is both necessary and sufficient for maximal fluorescence from $1 \mathrm{~J}$-aggregate. The increase in fluorescence between 5 to $20 \%$ methanol might be due to increased solubility of the cyanine in the solvent, thus enhancing J-aggregate formation. On the other hand, higher concentrations of methanol inhibited the self-assembly by disrupting the hydrophobic interactions between the chromophore and the scaffold. It is also possible that at high concentrations of methanol, the nucleic acids might be coming out of solution, thereby disrupting or decreasing the amount of $\mathrm{J}$-aggregate, resulting in fluorescence loss. This manifested in the biphasic curve shown in Fig. 4. The concentration of methanol to effect $50 \%$ reduction in fluorescence intensity $\left(\mathrm{IC}_{50}\right)$ was approximately the same for all four nucleic acid scaffolds, being $30-35 \%(\mathrm{v} / \mathrm{v})$.

With the exception of RNA, salt uniformly inhibited selfassembly and decreased the fluorescence intensity (Fig. 5). Unlike methanol profiles (Fig. 4), the strength of $\mathrm{NaCl}$ inhibition was different with different scaffolds (Fig. 5). Interaction between $\mathbf{1}$ and $\lambda \mathrm{DNA}$ was most sensitive to ionic strength, whereas interactions of $\mathbf{1}$ with the other three nucleic acid scaffolds were more resistant to salt (Fig. 5). The $\mathrm{NaCl}$ concentrations for $50 \%$ reduction in fluorescence intensity $\left(\mathrm{IC}_{50}\right)$ with the nucleic acid scaffolds were: $\lambda \mathrm{DNA}=10 \mathrm{mM} ; \phi \mathrm{X} 174$

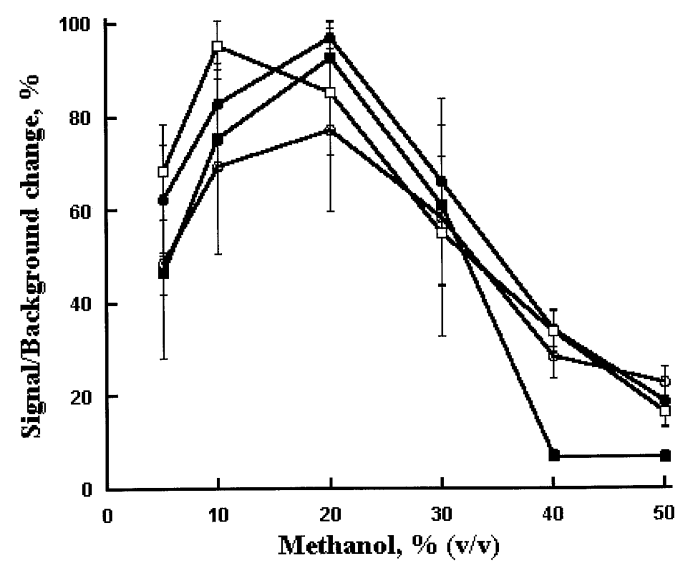

Fig. 4 Effects of methanol concentration on the self-assembly of cyanine 1. The tracing with open circles is $\phi \times 174$ DNA, closed circles $\lambda$ DNA, open squares Escherichia coli genomic DNA and closed squares Saccharomyces cerevisiae RNA.

DNA and genomic DNA $=100 \mathrm{mM}$; and RNA $=75 \mathrm{mM}$. The dose-response profile of $\phi$ X174 DNA (double stranded, circular DNA) and E. coli genomic DNA (linear, double stranded DNA) was nearly identical (Fig. 5). Salt effects could be rank-ordered as follows (least-to-most susceptible): $\phi$ X174 DNA = genomic DNA $>$ RNA $>\lambda$ DNA. The effect upon RNA was a departure from the rest of the nucleic acids, since it required $\sim 25 \mathrm{mM}$ $\mathrm{NaCl}$ for maximum fluorescence, declining thereafter. On the other hand, there was no stimulatory effect of $\mathrm{NaCl}$ upon the aggregation of 1 with the remaining nucleic acid scaffolds (Fig. 5).

The salt permits neutralization of the cationic charge density of the aggregate and allows electrostatic screening between the anionic nucleic acids and the cationic dye. ${ }^{14}$ Unlike earlier reports, ${ }^{16,17}$ we excluded a direct inhibitory effect of $\mathrm{NaCl}$ upon fluorescence, due to different perturbations of the ensembles (Fig. 5). Consistent with the data of Fang et al. ${ }^{17}$ some decrease in $S / B$ was due to an increase in intrinsic fluorescence of monomeric 1 in the presence of $\mathrm{NaCl}$. The salt and methanol sensitivities of $\lambda$ DNA suggested a self-assembly mechanism based upon electrostatic (coulombic) and hydrophobic forces. With the remaining nucleic acid scaffolds, both self-assembly and intercalation modes of interactions might be operative. Considering the effects of methanol and $\mathrm{NaCl}$ together, we speculate that non-covalent, reversible, and supramolecular selfassembly is the dominant mechanism without excluding a small extent of intercalation taking place between $\mathbf{1}$ and the various nucleic acids.

Changes in temperature and ionic strength were crucial for inter-conversion between fluorescent J-aggregate and nonfluorescent or minimally fluorescent $\mathrm{H}$-aggregate. ${ }^{14}$ As reported previously, cyanine exists as monomer, $\mathrm{H}$-aggregate and J-aggregate depending upon the temperature. Between the temperature range of 5 and $35^{\circ} \mathrm{C}$, cyanines formed $\mathrm{J}$-aggregates predominantly. ${ }^{14}$ Thermal stability of the J-aggregate was also dependent on the dye concentration. ${ }^{14}$ Since our experiments were conducted at $22^{\circ} \mathrm{C}$, within the window of J-aggregateforming temperatures, and due to the high dye-to-DNA ratio employed in our studies (vide infra), we believe that $\mathbf{1}$ was undergoing supramolecular self-assembly upon the nucleic acid scaffolds.

The full width at half maximum (FWHM) can be used to compare the spectral profiles obtained under different conditions. We obtained the fluorescence spectra for $\mathbf{1}$ selfassembling upon $\phi$ X174 DNA and carboxymethylamylose 


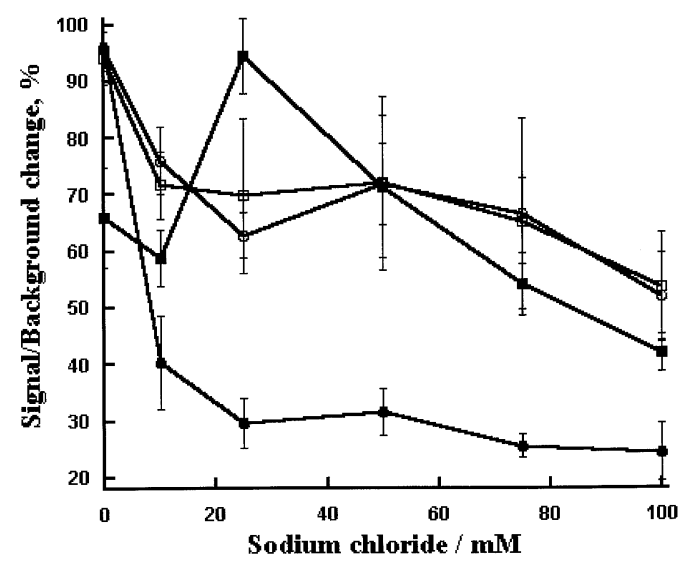

Fig. 5 Effects of salt concentration on the self-assembly of cyanine 1. The tracing with open circles is $\phi X 174$ DNA, closed circles $\lambda$ DNA, open squares Escherichia coli genomic DNA and closed squares Saccharomyces cerevisiae RNA.

(CMA) templates. Based upon spectroscopic and circular dichroism data, we previously demonstrated that supramolecular self-assembly was involved in the interaction of $\mathbf{1}$ and CMA. ${ }^{15}$ We found that the FWHM values for the fluorescence emission of 1-CMA and 1- $\phi$ X174 DNA ensembles were nearly identical $(\sim 19 \mathrm{~nm})$. Taken together, our data suggested that supramolecular self-assembly was involved in the interactions of $\mathbf{1}$ with nucleic acids.

\section{Cyanine 1 optimization for detecting nucleic acids}

The optimum concentration for detecting and quantizing $\phi X 174$ virion DNA was $2.5-5 \mu \mathrm{M}$ of $\mathbf{1}$ (Fig. 6). Similar biphasic trends were observed during dose-response titrations with the other nucleic acids, where the optimum concentration of 1 was $5-10 \mu \mathrm{M}$. This high dye-to-DNA is characteristic as reported previously ${ }^{8}$ and is similar to the $5 \mu \mathrm{M}$ concentration of a different cyanine optimized for detecting nucleic acids by Fang et al. ${ }^{17}$ As noted above, aggregation is influenced by several parameters including the type and concentration of the nucleic acid and the dye, ionic strength, $\mathrm{pH}$ and temperature. ${ }^{14}$ A biphasic curve was observed when 1 self-assembled upon hyaluronic acid. ${ }^{6}$ Two reasons might contribute to the decline in fluorescence with increasing concentrations of 1 (Fig. 6). The first is the "inner filter" effect arising from re-absorption of the emitted radiation perhaps by the scaffold. Inner filter effect may also arise from high concentrations of the absorbing chromophore. Therefore, the intensity of excitation light is not constant throughout the dye-DNA ensemble. Consequently, only a small portion of the excitation light reaches the ensemble, leading to diminished photon emission. A second possibility might be the perturbation of the J-aggregate at high ratios of $\mathbf{1}$ to scaffold. ${ }^{6}$ Between 100 to $500 \mu \mathrm{M}$ of $\mathbf{1}$, "self-absorption" (selfquenching) effect became prominent (Fig. 6).

\section{DNA quantization using cyanine 1}

We next constructed a calibration curve of $\phi X 174$ DNA with 5 $\mu \mathrm{M}$ of 1 (Fig. 7). We calculated limit of detection (LOD) of 3 fmol DNA and a limit of quantitation (LOQ) of 9 fmol (30 and $90 \mathrm{pM} ; 50$ and $150 \mathrm{ppb}$, respectively). Linearity of doseresponse and the overlap between data and curve fit were good (Fig. 7). The sensitivity for single stranded DNA were comparable to those reported using double stranded DNA, where detection limits are usually lower. ${ }^{18}$ Our assay can be miniaturized to ultra-high-throughput screening (uHTS) with

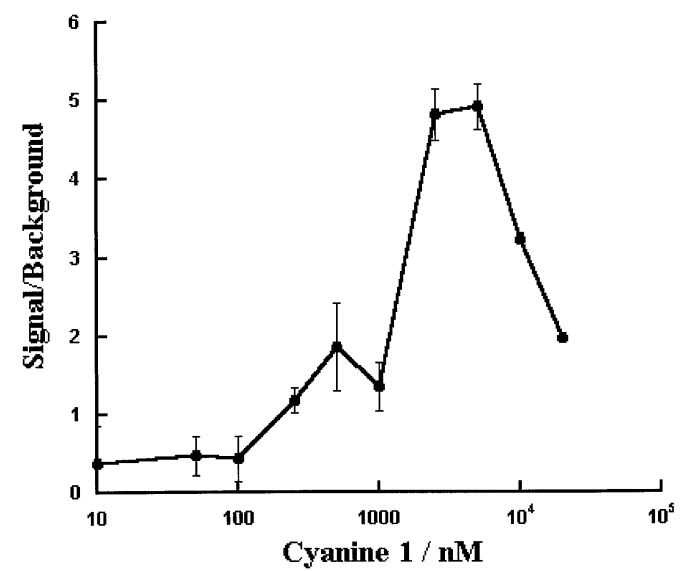

Fig. 6 Optimization of cyanine 1 concentration for the detection and quantization of $\phi \mathrm{X} 174 \mathrm{DNA}$. Increasing concentrations of $\mathbf{1}$ were mixed with $450 \mathrm{pM} \phi \mathrm{X} 174$ virion DNA in a total volume of 100 $\mu \mathrm{L}$ (45 fmol DNA). Other details are described under the Experimental section of the paper.

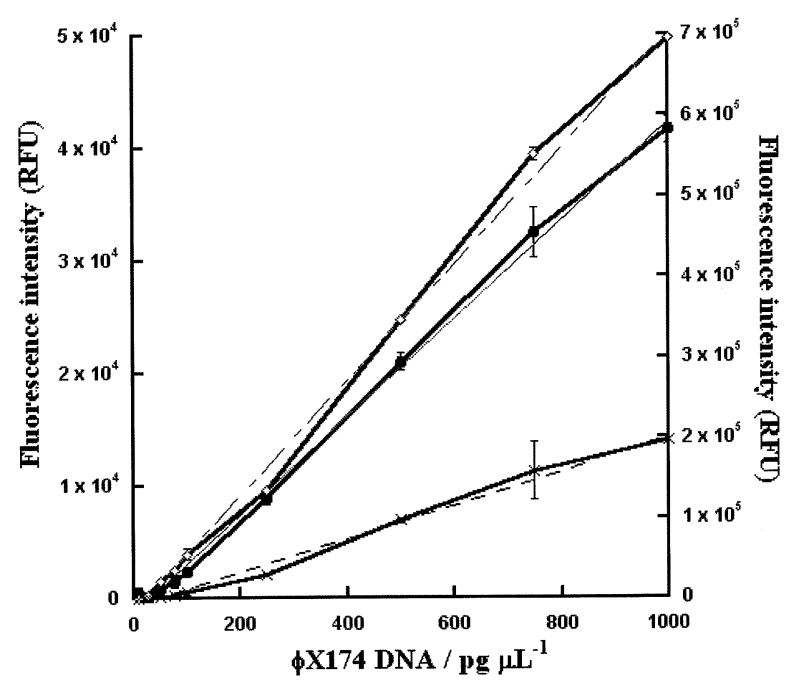

Fig. 7 Quantization of $\phi$ X174 DNA using the supramolecular selfassembly of cyanine 1. Increasing amounts of $\phi X 174$ DNA were reacted with $5 \mu \mathrm{M}$ 1. The closed circles data were collected with 355 $\mathrm{nm}$ (ex) and $460 \mathrm{~nm}$ (em) filters (left, $Y$-1 axis). The open diamonds data were obtained with $400 \mathrm{~nm}$ (ex) and $460 \mathrm{~nm}$ (em) filters (right, $Y-2$ axis). The cross symbols data were obtained using the monochromator-based instrument with $426 \mathrm{~nm}$ (ex) and $470 \mathrm{~nm}$ (em) (left, $Y-1$ axis). Linearity $\left(r^{2}\right)$ of the dose-response curves were: open diamonds, 0.99192; closed circles, 0.99830; crosses, 0.99675.

1536-wells using the principles described by us previously. ${ }^{12}$ The sensitivity for other nucleic acids ranged between 25 to 50 $\mathrm{ng} / \mathrm{mL}$ (LOD) and 75 to $150 \mathrm{ng} / \mathrm{mL}$ (LOQ), with the exception of RNA, where LOQ was $250 \mathrm{ng} / \mathrm{mL}$. Whereas 1 was equally sensitive toward single stranded, double stranded, linear and circular DNA, it was less sensitive for RNA. The reasons for this may relate to structural differences of the chromophore and/ or the scaffold (vide supra).

As summarized by Skripinets et al. ${ }^{19}$ PicoGreen was the most sensitive with a detection limit of $0.25 \mathrm{ng} / \mathrm{mL}$ DNA, followed by ethidium bromide $(10 \mathrm{ng} / \mathrm{mL})$, methylene blue $(30 \mathrm{ng} / \mathrm{mL})$, and finally, $\mathrm{Tb}(\mathrm{III})-1,10$-phenanthroline $(100 \mathrm{ng} / \mathrm{mL})$. A microplate assay utilizing SYBR Green I reported an LOD of $2000 \mathrm{ng} / \mathrm{mL}$ for $\lambda \mathrm{DNA}^{20}$ relative to our LOD of $25-50 \mathrm{ng} / \mathrm{mL}$. 


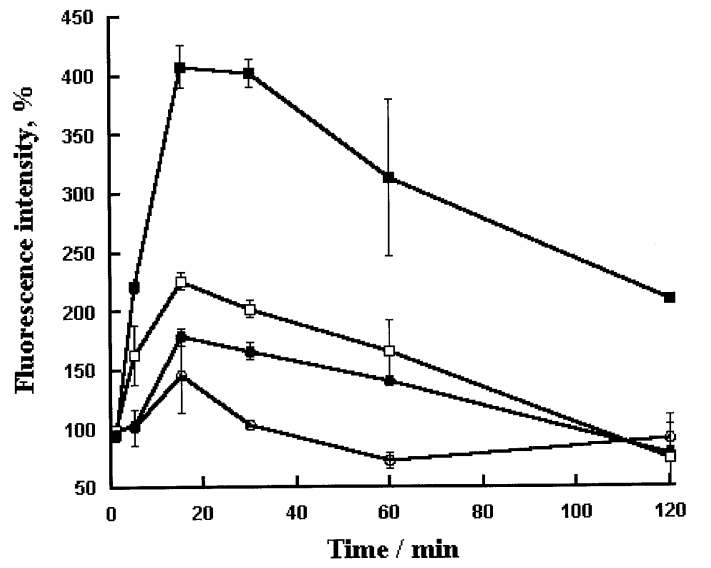

Fig. 8 Kinetics of the supramolecular self-assembly of cyanine $\mathbf{1}$ Cyanine 1 was mixed with $S$. cerevisiae RNA (closed squares), $\phi X 174$ DNA (open circles), $\lambda$ DNA (closed circles) and E. coli genomic DNA (open squares). Changes to the fluorescence intensity were measured over time.

Huang et al. ${ }^{21}$ reported LOD of 13 to $60 \mathrm{ng} / \mathrm{mL}$ for calf thymus DNA, fish sperm DNA and yeast RNA. They also reported LOD of $84-99 \mathrm{ng} / \mathrm{mL}$ for the same nucleic acids while using a copper-phenol method. ${ }^{22}$ More recently, Fang et al.,${ }^{17}$ reported LOD of $3-3.5 \mathrm{ng} / \mathrm{mL}$ using a cyanine dye to detect DNA by light scattering. These reports constitute the benchmarks for our assay.

\section{Self-assembly kinetics and photostability}

Results from the reaction wells that were repeatedly excited and wells that were dedicated for each time point were similar. However, due to a doubling of the background during multiple excitation/emission cycles, the $S / B$ was reduced. In order to segregate the photostability of the dye and the ensemble, only the data obtained from reaction wells that were interrogated for a single time point was used for analysis. Fluorescence was detected immediately upon mixing dye and the scaffold $(<1$ min) (Fig. 8). All the scaffolds required at least $15 \mathrm{~min}$ for optimum self-assembly. Highest increase in $S / B$ was noted for RNA resulting in nearly 4-fold brighter fluorescence. Other nucleic acid scaffolds showed an increase in $S / B$ over the $15 \mathrm{~min}$ window, albeit to lower extents. After $15 \mathrm{~min}$, there was a progressive decline in the emission, resulting in a biphasic temporal curve (Fig. 8). At 2 h, DNA-dye ensemble fluorescence dropped steeply reaching levels that were less than the initial fluorescence. By contrast, at $2 \mathrm{~h}$ the RNA-dye ensemble was twice as bright compared to $t=1 \mathrm{~min}$. These data are indicative of the relative stabilities of the various ensembles and hint at the assembly-disassembly processes taking place during dye-nucleic acid interactions. The increase in fluorescence intensity over time from the ensembles was rank ordered as follows: RNA $>$ genomic DNA $>\lambda$ DNA $>\phi X 174$ DNA (Fig. 8).

\section{Conclusions}

In summary, we describe the supramolecular self-assembly of a highly fluorescent cyanine J-aggregate upon various nucleic acid scaffolds. We describe several factors driving the assembly and dis-assembly of the cyanine. We present evidence for the noncovalent, reversible, electrostatic (coulombic) and hydrophobic interactions that are involved in supramolecular self-assembly. We used the fluorescence from the J-aggregate to detect and quantize various types of nucleic acids. We suspect that the binding of cyanine to the various nucleic acid scaffolds involves subtle changes to the conformation of the nucleic acids and also the existence of a competition between the chromophore and other nucleic acid-binding ligands such as polyamines. ${ }^{23}$ Studies are in progress to investigate these aspects modulating the selfassembly.

\section{Acknowledgements}

Sandia is a multi-program laboratory operated by Sandia Corporation, a Lockheed Martin Company, for the United States Department of Energy under Contract DE-AC04-94AL85000. K. A. thanks the Defense Threat Reduction Agency-Joint Science and Technology Office (DTRA-JSTO, contract \#AA07CBT008) for partially funding these investigations. This work was also supported in part by Sandia's Laboratory Directed Research and Development (LDRD) project \#105874 awarded to D. B. We thank Prof. Bruce Armitage, Department of Chemistry, Carnegie Mellon University, Pittsburgh, PA, for valuable comments.

\section{References}

1. J.-M. Lehn, Science, 2002, 295, 2400.

2. J.-M. Lehn, Proc. Natl. Acad. Sci. U. S. A., 2002, 99, 4763.

3. G. M. Whitesides and M. Boncheva, Proc. Natl. Acad. Sci. U. S. A., 2002, 99, 4769.

4. C. M. Niemeyer, Angew. Chem., Int. Ed., 2001, 40, 4128.

5. D. G. Whitten, K. E. Achyuthan, G. P. Lopez, and O.-K. Kim, Pure Appl. Chem., 2006, 78, 2313.

6. K. E. Achyuthan, L. Lu, G. P. Lopez, and D. G. Whitten, Photochem. Photobiol. Sci., 2006, 5, 931.

7. K. C. Hannah and B. A. Armitage, Acc. Chem. Res., 2004, 37, 845 .

8. B. A. Armitage, Top. Curr. Chem., 2005, 253, 55.

9. A. Mishra, R. K. Behera, P. K. Behera, B. K. Mishra, and G. P. Behera, Chem. Rev., 2000, 100, 1973.

10. T. Kobayashi, "J-Aggregates", 1996, World Scientific, Singapore.

11. L. Lu, R. M. Jones, D. McBranch, and D. Whitten, Langmuir, 2002, 18, 7706.

12. K. E. Achyuthan and D. G. Whitten, Comb. Chem. High Throughput Screening, 2007, 10, 399.

13. K. E. Achyuthan, Luminescence, 2001, 16, 257.

14. M. Wang, G. L. Silva, and B. A. Armitage, J. Am. Chem. Soc., 2000, 122, 9977.

15. O.-K. Kim, J. Je, G. Jernigan, L. Buckley, and D. Whitten, J. Am. Chem. Soc., 2006, 128, 510.

16. J. Xiang, X. Yang, C. Chen, Y. Tang, W. Yan, and G. Xu, J. Colloid Interface Sci., 2003, 258, 198.

17. F. Fang, H. Zheng, L. Li, Y. Wu, J. Chen, S. Zhou, and C. Zhu, Spectrochim. Acta, 2006, 64, 698.

18. K. Rengarajan, S. M. Cristol, M. Mehta, and J. M. Nickerson, Mol. Vision, 2002, 8, 416.

19. Y. V. Skripinets, A. V. Egorova, I. V. Ukrainets, and V. P. Antonovich, J. Anal. Chem., 2006, 61, 44.

20. J. Leggate, R. Allain, L. Isaac, and B. W. Blais, Biotechnol. Lett., 2006, 28, 1587.

21. C. Z. Huang, Y.-F. Li, and X. D. Liu, Anal. Chim. Acta, 1998, 375, 89.

22. Y.-M. Hao, Z.-X. Guo, X.-X. Wang, and H.-X. Shen, Anal. Chim. Acta, 1999, 402, 21.

23. V. Vijayanathan, T. Thomas, A. Shirahata, and T. J. Thomas. Biochemistry, 2001, 40, 13644. 